\title{
OBSERVAÇÃO DE RESISTÊNCIA PARCIAL A Septoria glycines EM SOJA
}

\author{
ÁLVARO M. R. ALMEIDA*
}

Embrapa Soja, Cx. Postal 231, 86001-970, Londrina, PR, e-mail: almeida@cnpso.embrapa.br

(Aceito para publicação em 12/03/2001 )

ALMEIDA, A.M.R. Observação de resistência parcial a Septoria glycines em soja. Fitopatologia Brasileira 26:214-216. 2001.

\section{RESUMO}

Seleção de genótipos de soja (Glycine max) resistentes à mancha parda foi avaliada em condições de casa de vegetação, inoculando-se plantas de soja com um mês de idade com suspensão de esporos calibrada para $10^{6}$ esporos $/ \mathrm{ml}$. A avaliação foi iniciada duas semanas após a inoculação e consistiu em determinar a área abaixo da curva de progresso de lesão, índice de amarelecimento e período necessário para atingir 5\% de área foliar necrosada. Nenhum genótipo testado foi imune à mancha parda da soja. No entanto, as cultivares CTS-40, IAS-2, IAS-5, PI PI 230975 e PI 204332 exibiram menor porcentagem de área foliar necrosada e apresentaram maior período em dias para atingir $5 \%$ de severidade. Os resultados mostram que o nível de severidade estabelecido baseado no número de dias para atingi-lo é um bom indicador para seleção de genótipos com resistência parcial a Septoria glycines. Os genótipos identificados como tolerantes, neste trabalho, poderão ser utilizados nos programas de melhoramento de soja no Brasil.

Palavras-chave: Glycine max, mancha parda, seleção de genótipos.

\section{ABSTRACT \\ Partial resistance to Septoria glycines in soybean}

Screening of soybean (Glycines max) genotypes resistant to brown spot was performed under greenhouse conditions by inoculating one-month-old plants with a suspension of spores, calibrated to $10^{6}$ spores $/ \mathrm{ml}$. Evaluation was based on the area under the lesion progress curves, level of chlorosis, and period to reach $5 \%$ severity, starting two weeks after inoculation. No cultivar was resistant to brown spot. However, cultivars
CTS-40, IAS-2, IAS-5, PI 230 975, and PI 204332 exhibited less infected leaf area and a longer period to reach $5 \%$ disease severity. The results showed that the level of severity established based on the number of days to reach it is a good indicator for screening soybean genotypes with partial resistance to $S$. glycines. Genotypes identified as tolerant in this work may be used in soybean breeding programs in Brazil.
A cultura da soja (Glycine max L.) é considerada como uma das mais importantes no Brasil, principalmente pela extensão da área cultivada com essa leguminosa. A produtividade brasileira em 1999 foi de $2367 \mathrm{~kg} / \mathrm{ha}$ (CONAB). No entanto, dados experimentais consideram que essa quantidade pode ser superior a $3200 \mathrm{Kg} / \mathrm{ha}$ caso se consiga reduzir o efeito das doenças.

Entre as doenças mais importantes da soja está a mancha parda, causada pelo fungo Septoria glycines Hemmi., descrito no Brasil em 1972 por Luzzardi et al. (1972). Estudos experimentais provaram que essa doença poderia causar danos de até 30\% (Almeida, 1980a). Dados recentes oriundos de avaliações nas principais regiões sojícolas do Brasil mostram que os prejuízos à cultura chegam a 315 milhões de dólares (Wrather et al., 1997).

A doença é inicialmente observada nas folhas primárias, causando lesões circulares, de cor vinho, que posteriormente tornam-se necróticas. É uma das primeiras doenças a aparecer nos campos de cultivo da soja. Normalmente a doença manifesta-se com maior intensidade a partir

\footnotetext{
* Bolsista do CNPq
}

do estádio R5 (início de formação de vagens), associando-se mais tarde à infecção por Cercospora kikuchii (Mat. \& Tomoy.) Gardner. No entanto, em períodos de seca, pode haver aumento de desfolha, especialmente de folhas localizadas no terço inferior das plantas.

O controle ideal da mancha parda seria através de genótipos resistentes. Infelizmente, nenhum genótipo com essa característica foi encontrado em soja (Lim, 1979). No entanto, diferenças em suscetibilidade, entre genótipos, foram observadas por Athow (1973). Há relatos, entretanto, que demonstram resistência nas espécies $G$. clandestina Wendl e G. tabacina (Labill.) Benth. (Lim \& Hymowitz,1987).

No Brasil, Almeida (1980b) observou que, em casa de vegetação, certos genótipos apresentavam diferenças quanto ao período de incubação e quanto à intensidade de sintomas foliares e níveis de amarelecimento do limbo foliar.

Este trabalho procurou selecionar genótipos de soja que apresentavam períodos mais longos para atingir 5\% de área foliar necrosada e menores porcentagens de área foliar lesionadas, quando inoculados artificialmente com $S$. glycines, em condições de casa de vegetação.

Oito genótipos, previamente selecionados quanto ao 
Observação de resistência parcial a Septoria glycines em soja

grau de suscetibilidade (Almeida, 1980b), foram semeados em vasos com solo esterilizado e mantidos em casa de vegetação, com temperatura média de $28{ }^{\circ} \mathrm{C} \pm 3{ }^{\circ} \mathrm{C}$. Utilizaram-se três vasos com quatro plantas por vaso. As plantas foram inoculadas quando apresentavam a segunda folha trifoliolada com metade de seu desenvolvimento, utilizandose $1 \mathrm{ml}$ de suspensão de esporos calibrada com hemacitômetro, para concentração final de $10^{6}$ esporos/ml (Almeida, 1980a). A inoculação foi feita através de atomizador acoplado a compressor com pressão de $4 \mathrm{Kgf} / \mathrm{cm}^{2}$. Imediatamente após a inoculação os vasos foram cobertos com filme plástico e posicionados sobre colchão de espuma encharcada com água, o qual manteve o ambiente saturado de umidade. Após $48 \mathrm{~h}$ retirou-se a cobertura plástica e a espuma.

O inóculo foi produzido em meio de BDA conforme descrito por Almeida (1978). Duas semanas após a inoculação iniciaram-se as avaliações da severidade da doença. Foram avaliadas a percentagem de área foliar necrosada, índice de amarelecimento ao redor das lesões e período de incubação, aqui utilizado como o número de dias para que as lesões atingissem $5 \%$ da área foliar inoculada e quando os sintomas começam a ser mais visíveis. A porcentagem de área necrosada foi feita por comparação e interpolação de valores utilizando-se escala diagramática descrita por James (1971). $\mathrm{O}$ índice de amarelecimento foi estabelecido arbitrariamente, sendo $1=$ ausência de amarelecimento; $2=$ amarelecimento restrito ao redor das lesões, com margens não superiores a $3 \mathrm{~mm} ; 3=$ até $25 \%$ de amarelecimento do limbo foliar; $4=>$ $25 \%$ do limbo foliar amarelecido.

Dados originais, obtidos em porcentagem de área foliar lesionada, foram usados para obtenção das áreas abaixo das curvas de progresso da lesão (AACPL), mediante cálculo de áreas trapezoidais obtidas pelas curvas de progresso de doença, em função do tempo em dias, nos diferentes genótipos, conforme descrição de Shaner \& Finney (1977).

Análises de variância foram utilizadas para verificar o efeito de tratamento e para comparar as médias da AACPL através do teste de Tukey, a 5\% de probabilidade. O mesmo método foi utilizado para comparar as médias dos períodos de incubação. Os experimentos foram repetidos duas vezes, utilizando-se os mesmos genótipos.

Todos os genótipos testados foram suscetíveis ao fungo. No entanto, observou-se diferenças significativas $(\mathrm{P}<5 \%)$ para os três parâmetros avaliados indicando que certos genótipos foram mais suscetíveis. A análise de variância, feita com os dados de AACPL, permitiu identificar que os genótipos CTS-40, IAS-2, IAS-5, PI 230975 e PI 204332 apresentaram resistência parcial à mancha parda da soja (Tabela 1). Da mesma forma, os genótipos com menor AACPL, também apresentaram períodos mais longos para atingir o nível de $5 \%$ de severidade, bem como, os menores valores do índice de amarelecimento (Tabela 1).

O fato de genótipos menos suscetíveis apresentarem maiores períodos para atingirem o nível de 5\% de severidade não foi observado por Lim (1979) ou por Athow (1973), provavelmente porque o objetivo era identificar imunidade
TABELA 1 - Área abaixo da curva de progresso de lesão (AACPL), período médio em dias para atingir severidade de $5 \%$ (PMS $5 \%$ ) e, índice de amarelecimento (IA) observados em genótipos de soja infetados com Septoria glycines*

\begin{tabular}{lccc}
\hline \hline Gentipo & AACPL & PMS (5\%) & IA** \\
\hline PI 227 221 & $875,43 \mathrm{a}$ & $17,00 \mathrm{a}$ & $4,0 \mathrm{a}$ \\
PI 224 270 & $775,95 \mathrm{a}$ & $17,33 \mathrm{a}$ & $3,5 \mathrm{a}$ \\
Davis & $775,12 \mathrm{a}$ & $20,33 \mathrm{a}$ & $3,5 \mathrm{a}$ \\
PI 204 332 & $450,20 \mathrm{~b}$ & $14,33 \mathrm{a}$ & $1,5 \mathrm{~b}$ \\
PI 200 509 & $446,52 \mathrm{~b}$ & $15,67 \mathrm{a}$ & $2,0 \mathrm{~b}$ \\
CTS - 40 & $383,40 \mathrm{~b}$ & $15,83 \mathrm{a}$ & $1, .0 \mathrm{~b}$ \\
IAS - 2 & $399,75 \mathrm{~b}$ & $33,17 \mathrm{~b}$ & $1,0 \mathrm{~b}$ \\
IAS - 5 & $391,86 \mathrm{~b}$ & $31,33 \mathrm{~b}$ & $1,0 \mathrm{~b}$ \\
\hline D.M.S.(5\%) & $\mathbf{1 0 0 , 6 7}$ & $\mathbf{8 , 1 2 ~ b}$ & $\mathbf{0 , 5 8}$ \\
\hline C.V. & $\mathbf{1 1 , 8 5}$ & $\mathbf{2 0 , 2 5}$ & $\mathbf{1 2 , 2 2}$ \\
\hline
\end{tabular}

* Valores correspondem à média de dois experimentos. Médias seguidas pela mesma letra, nas colunas, não diferem entre sí pelo teste de Duncan a 5\% de probabilidade.

** $1=$ trifólios sem amarelecimento; $2=$ lesões com bordas amarelecidas, com até $3 \mathrm{~mm}$ de largura; $3=$ até $25 \%$ da área foliar amarelecida; $4=>25 \%$ de área foliar amarelecida.

ou devido à diferença entre os genótipos utilizados.

Observações de estudos conduzidos anteriormente (Almeida, 1980b) mostraram que há casos de genótipos bastante afetados quanto a percentagem de área foliar necrosada mas que apresentam baixa percentagem de área foliar amarelecida: Aksarben (preta), PI 192.873, CTS-4, Biloxi e FC 31-750. O amarelecimento é comprovadamente causado por uma toxina hospedeiro-específica produzida por S. glycines (Song et al., 1993).

Constatou-se, neste trabalho, a ocorrência de diferentes níveis de suscetibilidade entre genótipos de soja, observada com o método de inoculação utilizado. Métodos de cruzamento específicos, como seleção recorrente, poderão utilizar essa resistência para obtenção de genótipos com maior nível de resistência, especialmente com genótipos já adaptados às condições brasileiras como IAS-2 e IAS-5.

\section{AGRADECIMENTOS}

O autor expressa seus agradecimentos a L. C. Benato pelo auxílio na inoculação e avaliação dos genótipos testados, aos Drs. Lilian Amorim e Armando Bergamin Filho ESALQ, aos colegas revisores da Fitopatologia Brasileira pelas críticas e sugestões; ao Dr. Carlos A. Arrabal Arias, pela discussão dos resultados.

\section{REFERÊNCIAS BIBLIOGRÁFICAS}

ALMEIDA, A.M.R. Ação de diferentes temperaturas e regimes de luz sobre a germinação dos esporos de Septori glycines Hemmi. Fitopatologia brasileira 3:211-214. 
1978.

ALMEIDA, A.M.R. Efeito da inoculação de Septoria glycines Hemmi em plantas de soja em quatro estádios de desenvolvimento. Fitopatologia brasileira 5:163-168. 1980a.

ALMEIDA, A.M.R. Reação de cultivares e linhagens de soja a Septoria glycines. Resultados de Pesquisa. Embrapa. 1980b.

ATHOW, K.L. Fungal diseases. In: Caldwell, B.E. (Ed.) Soybeans: Improvement, production and uses. Capítulo 13. American Soc. Agronomy, Madison, USA. 1973. pp. 459-489.

CONAB. Indicadiores da agropecuária. Ano VIII, No 12. Dezembro, 1999.

JAMES, C.A Manual of assessment keys for plant diseases. APS Press. St. Paul, MN. 1971.

LIM, S.M. Evaluation of soybean for resistance to Septoria brown spot. Plant Disease Report 63:242-245. 1979.
LIM, S.M. \& HYMOWITZ, T. Reactions of perennial wild species of genus Glycine to Septoria glycines. Plant Disease 71:891-893. 1987.

LUZZARDI, G.C., KUHN, G.B., WETZEL, D.P., GASTAL, M.F. \& RAUP, C. Mancha castanha da soja. Uma doença no Brasil. IPEAS. Indicação de pesquisa 8:1-3. 1972.

SHANER, G. \& FINNEY, R.E. The effect of nitrogen fertilization on the expression of slow-mildewing resistance in Knox wheat. Phytopathology 67:1051-1056. 1977.

SONG, H.S., LIM, S.M. \& CLARK, J.M. Purification and partial characterization of a host specific pathotoxin from culture filtrates of Septoria glycines. Phytopathology 83:659-661. 1993.

WRATHER, J.A., ANDERSON, T.R., ARSYAD, D.M., PLOPER, L.D., PORTA-PUGLIA, A., RAM, H.H. \& YORINORI, J.T. Soybean disease losses estimates for the top 10 soybean producing countries in 1994. Plant Disease 78:107-110. 1997. 